\title{
Interacting Tachyon: generic cosmological evolution for a tachyon and a scalar field
}

\author{
A. de la Macorra and U. Filobello \\ Instituto de Física, \\ Universidad Nacional Autónoma de México, \\ Apdo. Postal 20-364, 01000 D.F. México
}

\begin{abstract}
We study the cosmological evolution of a tachyon scalar field $T$ with a Dirac-Born-Infeld type lagrangian and potential $V(T)$ coupled to a canonically normalized scalar field $\phi$ with an arbitrary interaction term $B(T, \phi)$ in the presence of a barotropic fluid $\rho_{b}$, which can be matter or radiation. The force between the barotropic fluid and the scalar fields is only gravitational. We show that the dynamics is completely determine by only three parameters $\lambda_{1}=-V_{T} / V^{3 / 2}, \lambda_{2}=-B_{T} / B^{3 / 2}$ and $\lambda_{3}=-B_{\phi} / B$. We determine analytically the conditions for $\lambda_{i}$ under which the energy density of $T, \phi$ and $\rho_{b}$ have the same redshift. We study the behavior of $T$ and $\phi$ in the asymptotic limits for $\lambda$ and we show the numerical solution for different interesting cases.

The effective equation of state for the tachyon field changes due to the interaction with the scalar field and we show that it is possible for a tachyon field to redshift as matter in the absence of an interaction term $B$ and as radiation when $B$ is turned on. This result solves then the tachyonic matter problem.
\end{abstract}

\section{INTRODUCTION}

String tachyon fields $T$ are the lowest energy state in unstable Dp-brane or brane-antibrane systems [1]. Since they represent the low energy limit of string-brane models the phenomenology of the tachyon field is important and a great amount of work has been invested in studying the dynamics of tachyon field [1],[2]. In the case of a Dp-brane systems in string theory, the potential $V(T)$ has been conjectured to be tachyonic at the origin $T=0$ [1], i.e. the potential has a maximum at the origin with a negative mass square, $m^{2}=V_{T T}<0$.

The tachyon field has a Dirac-Born-Infeld type lagrangian and therefore it does not have canonical kinetic terms. This implies that the naive prescription for the mass of tachyon as the $m^{2}=V_{T T}$ does not hold in this case [5] nor can we assume that the evolution of $T$ is to reach the minimum of the potential $V(T)$, as for a standard scalar field, since $V(T)$ does not correspond to the true potential for $T$.

It is well known that the tachyon field has an equation of state parameter $-1 \leq w \leq 0$ [2]. For string motivated potentials, e.g. $V \simeq e^{-T^{2} / 2}$, the late time behavior gives $w=0$ and $T$ behaves as matter. This is the "matter problem" because $T$ can easily dominate the universe well before radiation-matter equality since it redshifts slower than radiation. So, if $T$ is present at early times it should necessarily decay into other particles to avoid the matter problem.

In this letter we study the generic cosmological evolution of a scalar field $T$ with a Dirac-Born-Infeld type lagrangian coupled to a canonical scalar field through an arbitrary interaction term $B(T, \phi)$ in the presence a barotropic fluid, which can be matter or radiation. We will call the field $T$ as the tachyon field even though we do not constrain ourselves to a potential $V(T)$ with $m^{2}<0$ at the origin, so our results are valid for any potential $V(T)$. We show that all models dependence is given in terms of three parameters $\lambda_{1}=-V_{T} / V^{3 / 2}, \lambda_{2}=-B_{T} / B^{3 / 2}$ and $\lambda_{3}=-B_{\phi} / B$. We determine the dynamical equations and obtain the attractor solutions as a function of these $\lambda_{i}$ parameters. We show in this letter that it is possible for a tachyon field to redshift as matter in the absence of an interaction term $B$ and to redshift at late times as radiation due to the interaction term. Therefore, the interaction term solves the tachyonic matter problem.

This letter is organized as follows. In section II we set up the framework for the cosmological evolution of two scalar fields, a tachyon and a canonical scalar field, with an arbitrary potential in the presence of a barotropic fluid. In section III we derive the dynamical first order differential equations and we show that the system is determined by only three parameters. In section IV we calculate the critical points. In section V we study different asymptotic limits and we present a discussion on specific particle physics motivated examples in section VA. Finally in section VB we give some interesting examples and we present our conclusions in section VI.

\section{COUPLED TACHYON AND SCALAR FIELD}

Our starting point is a universe filled with two scalar fields $T, \phi$ and a barotropic energy density $\rho_{b}$, which can be either matter $w_{b}=0$ or radiation $w_{b}=1 / 3$. We will assume that the scalar fields interact via a potential $B(T, \phi)$ while there is only gravitational interaction between these fields and the barotropic fluid. This work generalizes that 
of a single scalar field and a barotropic fluid [3], a tachyon field and a barotropic fluid[6] and two single scalar field with arbitrary potential and a barotropic fluid [7].

One of this scalar fields, namely $T$, is a tachyon field which is motivated by string theory and D-branes, while the other scalar field $\phi$ is a standard canonical field. We take the following Lagrangian for the scalar fields $\phi$ and $T$ [1]

$$
L=-V(T) \sqrt{1-\partial_{\mu} T \partial^{\mu} T}+\frac{1}{2} \partial_{\mu} \phi \partial^{\mu} \phi-B(\phi, T)
$$

where the tachyon is given by a Dirac-Born-Infeld type lagrangian with a potential $V(T)$ and $B(\phi, T)$ corresponds to the interaction between $\phi$ and the tachyon $T$. The potentials $V(T)$ and $B(\phi, T)$ are completely arbitrary. The equation of motion of $\phi$ and $T$ for a spatially flat Friedman-Robertson-Walker (FRW) universe are

$$
\begin{aligned}
\ddot{\phi}+3 H \dot{\phi}+B_{\phi} & =0 \\
\frac{\ddot{T}}{1-\dot{T}^{2}}+3 H \dot{T}+\frac{V_{T}}{V}+\frac{B_{T}}{V} \sqrt{1-\dot{T}^{2}} & =0
\end{aligned}
$$

where the subindex in $V$ and $B$ is defined as $V_{T} \equiv \partial V / \partial T, B_{\phi} \equiv \partial B / \partial \phi$ and $B_{T} \equiv \partial B / \partial T$. The Hubble parameter $H \equiv \dot{a} / a$ is

$$
3 H^{2}=\rho=\rho_{\phi}+\rho_{T}+\rho_{b}
$$

where we have taken $8 \pi G \equiv 1$ and $\rho$ is the total energy density, $\rho_{b}$ the barotropic fluid and $\rho_{\phi}, \rho_{T}$ are defined as

$$
\rho_{\phi} \equiv \frac{1}{2} \dot{\phi}^{2}+V(\phi), \quad p_{\phi} \equiv \frac{1}{2} \dot{\phi}^{2}-V(\phi)
$$

for $\phi$ and

$$
\rho_{T} \equiv \frac{V}{\sqrt{1-\dot{T}^{2}}}, \quad p_{T} \equiv V \sqrt{1-\dot{T}^{2}}
$$

for $T$ with $p_{\phi}, p_{T}$ the pressure of $\phi, T$, respectively. We define the ratio of energy densities as $\Omega_{T} \equiv \rho_{T} / 3 H^{2}$, $\Omega_{\phi} \equiv \rho_{\phi} / 3 H^{2}$. Using eqs.(5) and (6) we can rewrite the dynamical eqs.(2) and (3) in terms of the energy densities as

$$
\begin{aligned}
\dot{\rho}_{\phi}+3 H \rho_{\phi}\left(1+w_{\phi}\right) & =B_{T} \dot{T}=\delta \\
\dot{\rho}_{T}+3 H \rho_{T}\left(1+w_{T}\right) & =-B_{T} \dot{T}=-\delta \\
\dot{\rho}_{b}+3 H \rho_{b}\left(1+w_{b}\right) & =0
\end{aligned}
$$

where we have included the evolution of the barotropic fluid $\rho_{b}$ and

$$
\delta \equiv B_{T} \dot{T}
$$

defines the interaction term. The equation of state parameters are given by

$$
w_{\phi} \equiv \frac{p_{\phi}}{\rho_{\phi}}=\frac{\frac{1}{2} \dot{\phi}^{2}-V}{\frac{1}{2} \dot{\phi}^{2}+V}, \quad w_{T} \equiv \frac{p_{T}}{\rho_{T}}=-1+\dot{T}^{2} .
$$

In order to have a real energy density for the tachyon we require $0 \leq \dot{T}^{2} \leq 1$ and from eq.(9) we see that the equation of state parameter for $T$ is constraint to $-1 \leq w_{T} \leq 0$. The time derivative of $H$ is given by

$$
\dot{H}=-\frac{1}{2}\left(\rho_{\phi}+\rho_{T}+\rho_{b}+p_{\phi}+p_{T}+p_{b}\right)=-\frac{1}{2}\left(\dot{\phi}^{2}+\rho_{T} \dot{T}^{2}+\rho_{b}\left(1+w_{b}\right)\right) .
$$

\section{A. Effective Equation of State}

From the last two terms in eq.(3) we can define a $T$-derivative of an effective potential $V_{\text {eff }}$ as

$$
\frac{d V_{e f f}}{d T} \equiv\left(1-\dot{T}^{2}\right)\left(\frac{V_{T}}{V}+\frac{B_{T}}{V} \sqrt{1-\dot{T}^{2}}\right)=\frac{V\left(V_{T}+B_{T} \sqrt{1-\dot{T}^{2}}\right.}{\rho_{T}^{2}}
$$


where we have used $\rho_{T}=V / \sqrt{1-\dot{T}^{2}}$. The dynamics of the tachyon field gives a vanishing $d V_{\text {eff }} / d T$, i.e. $d V_{e f f} / d T=$ $\left(V_{T}+B_{T} \sqrt{1-\dot{T}^{2}}\right) V / \rho_{T}^{2}=0$. It is important to include in eq.(11) the multiplicative factor $\left(1-\dot{T}^{2}\right)$. The dynamics of a scalar field is to minimize the potential (i.e. the derivative of the potential w.r.t. the scalar field should vanish) and in the absence of the interaction term $B$ the dynamics of the tachyon field does not give a vanishing $V_{T} / V$ but it has a vanishing $\left(1-\dot{T}^{2}\right) V_{T} / V$. For example for a typical tachyon potential $V \sim e^{-T^{2} / 2}$ one has $V_{T} / V=-T$ and the solution to eq.(3) gives $\dot{T}^{2}=1, T \rightarrow \infty$ with $V_{T} / V \rightarrow-\infty$ but $\left(1-\dot{T}^{2}\right) V_{T} / V=V_{T} V / \rho_{T}^{2}=0$. This shows that $V_{T} / V$ is not the $T$-derivative of the potential for the tachyon $T$.

At vanishing $d V_{\text {eff }} / d T$, eq.(3) becomes $\ddot{T}+3 H \dot{T}\left(1-\dot{T}^{2}\right)=-d V_{e f f} / d T=0$ and it has two solutions:

$$
V=0, \quad \ddot{T}=0, \quad \dot{T}^{2}=1
$$

and

$$
V_{T}=-B_{T} \sqrt{1-\dot{T}^{2}}, \quad \frac{\dot{T}}{\sqrt{1-\dot{T}^{2}}}=k a(t)^{-3}
$$

with $k$ an integration constant. The solution in eq.(12) necessarily implies $V=0$ since $\Omega_{T}=V /\left(3 H^{2} \sqrt{1-\dot{T}^{2}}\right)$ must be smaller than one while eq.(13) gives at late times $\dot{T}=0$ and $V_{T}=-B_{T}$.

In order to define an effective equation of state it is convenient to rewrite eqs.(7) as

$$
\dot{\rho}_{\phi}=-3 H \rho_{\phi}\left(1+w_{\phi e f f}\right), \quad \dot{\rho}_{T}=-3 H \rho_{T}\left(1+w_{T e f f}\right)
$$

with the effective equation of state defined by

$$
w_{\phi e f f} \equiv w_{\phi}-\frac{B_{T} \dot{T}}{3 H \rho_{\phi}}, \quad w_{T e f f} \equiv w_{b}+\frac{B_{T} \dot{T}}{3 H \rho_{T}} .
$$

We see from eqs.(14) that $w_{\phi e f f}, w_{T e f f}$ give the complete evolution of $\rho_{\phi}$ and $\rho_{T}$. For $B_{T} \dot{T}>0$ we have $w_{e f f}<w_{\phi}$ and the fluid $\rho_{\phi}$ will dilute slower than without the interaction term (i.e. $B_{T} \dot{T}=0$ ) while $\rho_{T}$ will dilute faster since $w_{\text {Teff }}<w_{\varphi}$. Which fluid dominates at late time will depend on which effective equation of state is smaller. The difference in eqs.(15) is [3]

$$
\Delta w_{e f f} \equiv w_{\text {Teff }}-w_{\phi e f f}=\Delta w-\Upsilon
$$

with $\Delta w \equiv w_{T}-w_{\phi}$ and $\Upsilon$ defined as

$$
\Upsilon=\frac{\delta}{3 H}\left(\frac{\rho_{\phi}+\rho_{T}}{\rho_{\phi} \rho_{T}}\right)=\frac{B_{T} \dot{T}}{3 H}\left(\frac{\rho_{\phi}+\rho_{T}}{\rho_{\phi} \rho_{T}}\right)=\frac{B_{T} \dot{T}}{9 H^{3}}\left(\frac{\Omega_{\phi}+\Omega_{T}}{\Omega_{\phi} \Omega_{T}}\right)
$$

while the sum gives

$$
\Omega_{T} w_{T e f f}+\Omega_{\phi} w_{\phi e f f}=\Omega_{T} w_{T}+\Omega_{\phi} w_{\phi}
$$

Clearly the relevant quantity to determine the relative growth is given by $\Upsilon$ and if $\Upsilon<\Delta w$ we have $\Delta w_{e f f}>0$ (i.e. $w_{\text {Teff }}>w_{\phi e f f}$ ) and $\rho_{\phi}$ will dominate the universe at late times with $\Omega_{\phi}=1, \Omega_{T}=0$. For $\Upsilon>\Delta w$ we have $\Delta w_{\text {eff }}<0$ and $w_{\text {Teff }}<w_{\phi e f f}$ with $\rho_{T}$ prevailing and $\Omega_{T}=1, \Omega_{\phi}=0$. If both fluids have the same redshift, i.e. $\Delta w_{\text {eff }}=0$ and $w_{\text {eff }} \equiv w_{\text {Teff }}=w_{\phi e f f}$ eq.(18) gives

$$
\frac{\Omega_{T}}{\Omega_{\phi}}=\frac{w_{\phi}-w_{e f f}}{w_{e f f}-w_{T}}
$$

and for $w_{\phi}<w_{T}$ we have

$$
w_{\phi} \leq w_{\phi e f f}=w_{T e f f}=\frac{w_{T} \Omega_{T}+w_{\phi} \Omega_{\phi}}{\Omega_{T}+\Omega_{\phi}} \leq w_{T},
$$

while for $w_{\phi}>w_{T}$ we get

$$
w_{\phi} \geq w_{\phi e f f}=w_{T e f f}=\frac{w_{T} \Omega_{T}+w_{\phi} \Omega_{\phi}}{\Omega_{T}+\Omega_{\phi}} \geq w_{T},
$$

i.e. the effective equation of state is constraint between $w_{\phi}$ and $w_{T}$. In the limit of no interaction $\delta=B_{\phi} \dot{\phi}=0$ we get $\Upsilon=0$ and $\Delta w_{\text {eff }}=\Delta w$ and depending in which term is smaller $w_{\phi}$ or $w_{T}$ we will have either $\rho_{\phi}$ or $\rho_{T}$ dominating the universe at late times. 


\section{B. Canonical Scalar Field}

In the limit of having only a canonical scalar field $\phi$ and a barotriopic fluid, the potential $B$ depends only on $\phi$. If the vacuum expectation value of $\phi_{\min }$ has a finite value, i.e. the potential is of the type $B=b_{o} \phi^{n}$, then the scalar field $\phi$ oscillates around its vacuum expectation value (v.e.v.). If the scalar field has a non zero mass or if the potential $B$ admits a Taylor expansion around $\phi_{\text {min }}$ then, using the Hôpital rule, one has $\lim _{t \rightarrow \infty}\left|B_{\phi} / B\right|=\infty$ and the energy density $\rho_{\phi}$ redshifts with $w_{\phi}=(n-2) /(n+2)$, i.e. $w_{\phi}=0,1 / 3$ for $n=2,4[3]$. On the other hand, if $\phi_{\min }=\infty$ then $\phi$ will not oscillate and $\left|B_{\phi} / B\right|$ will approach either zero, a finite constant or infinity. Only in the case $\left|B_{\phi} / B\right|$ going to zero or a constant smaller than $\sqrt{2}$ will the universe accelerate at late times [3].

\section{Tachyon Scalar Field}

If we have only a tachyon field in the presence of a barotropic fluid, the late time attractor solution were studied in [6]. A tracking solution with constant $\lambda_{1} \equiv-V_{T} / V^{3 / 2}$ is given for a potential $V=V_{o} / T^{2}$. In this case one finds $\dot{T}=y_{1} \lambda_{1} / \sqrt{3}$ and $y_{1}^{2}=V / 3 H^{2}=\left(\sqrt{\lambda_{1}^{4}+36}-\lambda_{1}^{2}\right) / 6$ with $w_{T}=-1+\dot{T}^{2}=-1+\left(\sqrt{\lambda_{1}^{4}+36}-\lambda_{1}^{2}\right) \lambda_{1}^{2} / 18$ if $w_{b}>w_{T}$ and $w_{T}=w_{b}$ if $w_{b}<-1+\left(\sqrt{\lambda_{1}^{4}+36}-\lambda_{1}^{2}\right) \lambda_{1}^{2} / 18$ with $\left(x_{1}, y_{1}\right)=\left(\gamma_{b}, \pm \sqrt{3 \gamma_{b}} / \lambda_{1}\right)$. [6]. Clearly a $\left|\lambda_{1}\right| \ll 1$ gives an equation of state $w_{T} \approx-1$ and an accelerating universe.

\section{GENERIC DYNAMICAL ANALYSIS}

To determine the attractor solutions of the differential equations given in eqs.(2) and (3) or (7) it is useful to make the following change of variables

$$
\begin{array}{ll}
x_{1} \equiv \dot{T}, & y_{1} \equiv \frac{1}{H} \sqrt{\frac{V(T)}{3}} \\
x_{2} \equiv \frac{\dot{\phi}}{\sqrt{6} H}, & y_{2} \equiv \frac{1}{H} \sqrt{\frac{B(T, \phi)}{3}}
\end{array}
$$

and eqs.(7) and (10) become a set of dynamical differential equations of first order

$$
\begin{aligned}
& x_{1 N}=-\left(1-x_{1}^{2}\right)\left(3 x_{1}-\sqrt{3} \lambda_{1} y_{1}-\sqrt{3\left(1-x_{1}^{2}\right)} \lambda_{2} \frac{y_{2}^{3}}{y_{1}^{2}}\right) \\
& y_{1 N}=-\frac{H_{N}}{H} y_{1}-\frac{\sqrt{3}}{2} \lambda_{1} x_{1} y_{1}^{2} \\
& x_{2 N}=-\left(3+\frac{H_{N}}{H}\right) x_{2}+\sqrt{\frac{3}{2}} \lambda_{3} y_{2}^{2} \\
& y_{2 N}=-\frac{H_{N}}{H} y_{2}-\frac{\sqrt{3}}{2} \lambda_{2} x_{1} y_{2}^{2}-\sqrt{\frac{3}{2}} \lambda_{3} x_{2} y_{2} \\
& \frac{H_{N}}{H}=-\frac{3}{2}\left(\Omega_{1} \gamma_{1}+\Omega_{2} \gamma_{2}+\Omega_{b} \gamma_{b}\right)=-\frac{3}{2}\left(x_{1}^{2} \Omega_{1}+2 x_{2}^{2}+\Omega_{b} \gamma_{b}\right)
\end{aligned}
$$

where $N$ is the logarithm of the scale factor $a, N \equiv \ln (a), \gamma_{b} \equiv 1+w_{b}, \gamma_{1} \equiv 1+w_{1}, \gamma_{2} \equiv 1+w_{2}$ and $f_{N} \equiv d f / d N$ for $f=H, x_{i}, y_{i}(i=1,2), \Omega_{b}=1-\Omega_{1}-\Omega_{2}$ and

$$
\lambda_{1}(N) \equiv-\frac{V_{T}}{V^{3 / 2}}, \quad \lambda_{2}(N) \equiv-\frac{B_{T}}{B^{3 / 2}}, \quad \lambda_{3}(N) \equiv-\frac{B_{\phi}}{B} .
$$

Notice that all model dependence in eqs.(24) is through the three quantities $\lambda_{i}(N), i=1,2,3$ and the constant parameter $\gamma_{b}=1+w_{b}$. The last equation of (24) is constraint between $-3 \leq H_{N} / H \leq 0$ for all values of $x_{i}, y_{i}$ and $\gamma_{b}$, it takes the value -3 when the universe is dominated by the kinetic energy $x_{2}^{2}=1$ and therefore $\Omega_{b}=\Omega_{1}=0$ while it becomes $H_{N} / H=0$ when $x_{2}=x_{1}=\Omega_{b}=0$ and the universe is dominate by a constant potential $y_{1}^{2}+y_{2}^{2}=1$. The set of equations given in eqs.(24) give the evolution of two scalar fields $\phi, T$, a tachyon and a canonical scalar field, with arbitrary potentials in the presence of a barotropic (perfect) fluid with equation of state $w_{b}=1-\gamma_{b}$. If we do not want to consider the contribution from the barotropic fluid we can easily take the limit $\gamma_{b}=0$ in eqs. $(25)$ 
since all contribution form $\rho_{b}$ is given in $H_{N} / H$ via the term $\Omega_{b} \gamma_{b}$. For $\Omega_{b} \neq 0$ we will assume a barotropic fluid with $0<\gamma_{b}<2$ and $\gamma_{b}=1$ for matter while $\gamma_{b}=4 / 3$ for radiation.

In terms of $x_{i}, y_{i}$ we have

$$
\begin{array}{lll}
\Omega_{T}=\Omega_{1}=\frac{\rho_{T}}{3 H^{2}}=\frac{y_{1}^{2}}{\sqrt{1-x_{1}^{2}}}, & \frac{p_{T}}{3 H^{2}}=-y_{1}^{2} \sqrt{1-x_{1}^{2}} \\
\Omega_{\phi}=\Omega_{2}=\frac{\rho_{\phi}}{3 H^{2}}=x_{2}^{2}+y_{2}^{2}, & \frac{p_{\phi}}{3 H^{2}}=x_{2}^{2}-y_{2}^{2}
\end{array}
$$

and

$$
w_{1} \equiv w_{T}=\frac{p_{T}}{\rho_{T}}=-1+x_{1}^{2}, \quad w_{2} \equiv w_{\phi}=\frac{p_{\phi}}{\rho_{\phi}}=\frac{x_{2}^{2}-y_{2}^{2}}{x_{2}^{2}+y_{2}^{2}}
$$

with $\gamma_{1}=1+w_{1}=x_{1}^{2}, \gamma_{2}=1+w_{2}=2 x_{2}^{2} / \Omega_{2}$. The interaction term defined in eq.(8) and (17) are now

$$
\begin{aligned}
\delta & =B_{T} \dot{T}=-3^{3 / 2} H^{3} \lambda_{2} x_{1} y_{2}^{3} \\
\Upsilon & =-\frac{\lambda_{2} x_{1} y_{2}^{3}}{\sqrt{3}}\left(\frac{\Omega_{1}+\Omega_{2}}{\Omega_{1} \Omega_{2}}\right)
\end{aligned}
$$

giving an effective equation of state parameters defined in eqs.(15) as

$$
\begin{aligned}
& w_{1 e f f} \equiv w_{\text {Teff }}=w_{1}-\frac{\lambda_{2} x_{1} y_{2}^{3}}{\sqrt{3} \Omega_{1}}=\frac{\sqrt{3} \Omega_{1} w_{1}-\lambda_{2} x_{1} y_{2}^{3}}{\sqrt{3} \Omega_{1}} \\
& w_{2 e f f} \equiv w_{\phi e f f}=w_{2}+\frac{\lambda_{2} x_{1} y_{2}^{3}}{\sqrt{3} \Omega_{2}}=\frac{\sqrt{3} \Omega_{2} w_{2}+\lambda_{2} x_{1} y_{2}^{3}}{\sqrt{3} \Omega_{2}}
\end{aligned}
$$

and

$$
\gamma_{1 e f f} \equiv 1+w_{1 e f f}, \quad \gamma_{2 \text { eff }} \equiv 1+w_{2 e f f}, \quad \gamma_{b e f f} \equiv \gamma_{b}
$$

The acceleration of the universe is given by

$$
\frac{\ddot{a}}{a}=H^{2}\left(1+\frac{H_{N}}{H}\right)=-\frac{H^{2}}{2}\left(\Omega_{b}\left(1+3 w_{b}\right)+\Omega_{1}\left(3 x_{1}^{2}-2\right)+4 x_{2}^{2}-2 y_{2}^{2}\right)
$$

where we have used $\dot{H}=H H_{N}$ and eq.(25). Clearly acceleration will occur if the universe is dominated by the potential $y_{2}^{2}=B / 3 H^{2}$ or $\Omega_{1}$ with $x_{1}^{2}<2 / 3$, i.e. for $y_{2}^{2}+\Omega_{1}\left(1-3 x_{1}^{2} / 2\right)>\Omega_{b}\left(1+3 w_{b}\right)+4 x_{2}^{2}$.

\section{CRITICAL SOLUTIONS}

To find the critical solutions to the dynamical equations (24) we need to solve them for $x_{1 N}=x_{2 N}=y_{1 N}=y_{2 N}=0$ for constant values of $\lambda_{i}, i=1,2,3$. However, the set of equations are highly non linear and it is quite complicated to find all critical points. Instead, we will calculate the critical points when all three fluids have the same redshift. We will also study different limits and we solve eqs.(24) numerically to show the behavior of the scalar fields $T$ and $\phi$.

As discussed in section II A the energy density $\rho_{1}, \rho_{2}, \rho_{b}$ with the smallest effective equation of state, or alternatively with the smallest $\gamma_{i \text { eff }}=1+w_{\text {ieff }}, i=1,2, b$, will dominate the universe. For $\gamma_{i \text { eff }}<\gamma_{k \text { eff }}, \gamma_{j \text { eff }}$ we have $\Omega_{i}=1$ and $\Omega_{k}=\Omega_{j}=0$ at late times. If two or the three energy densities have the same (smallest) effective equation of state then two $\Omega$ 's or all three $\Omega^{\prime} s$ will be different than zero. Therefore, eq.(25) gives asymptotically

$$
-\frac{2 H_{N}}{3 H}=\left(\Omega_{1} \gamma_{1}+\Omega_{2} \gamma_{2}+\Omega_{b} \gamma_{b}\right)=\left(\Omega_{1} \gamma_{1 \text { eff }}+\Omega_{2} \gamma_{2} \text { eff }+\Omega_{b} \gamma_{b}\right)=\gamma_{e f f}
$$

where the last equality in eq.(36) is valid only asymptotically and $\gamma_{e f f}=1+w_{e f f}$ is the smallest of the $\gamma_{e f f}$ 's, i.e. $\gamma_{\text {eff }}=\operatorname{smallest}\left(\gamma_{1 \text { eff }}, \gamma_{2 \text { eff }}, \gamma_{b}\right)$.

If one of the scalar fields dominates over the other one then eqs.(24) will be reduced to a single scalar field, either $T$ or $\phi$, in the presence of a barotropic fluid. These special case have been studied in the literature for tachyon domination [6] and for canonical scalar field in [3]. 

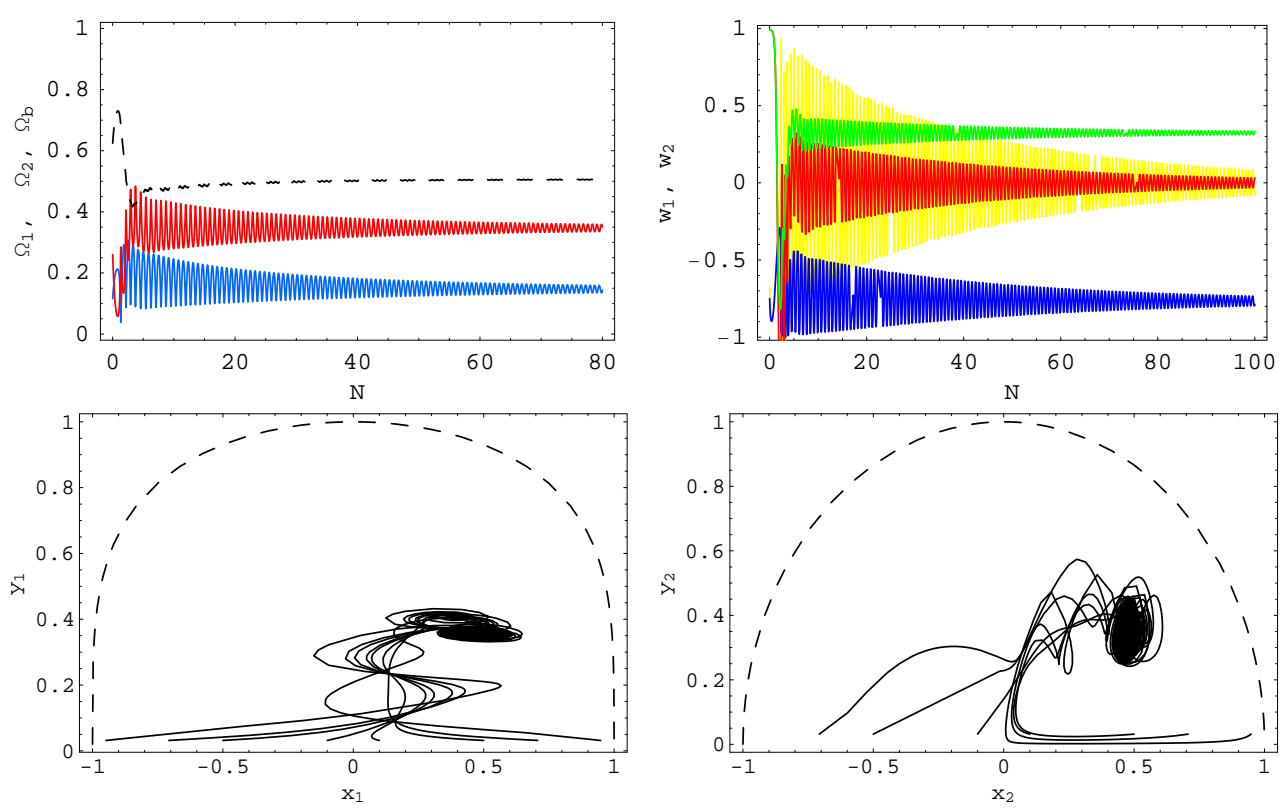

FIG. 1: We show for $\lambda_{1}=10, \lambda_{2}=-10, \lambda_{3}=5$ and $\gamma_{b}=1+w_{b}=1$ the evolution of $\Omega_{1}=\Omega_{T}, \Omega_{2}=\Omega_{\phi}, \Omega_{b}$ (blue (solid), red (dotted) and black (dashed), respectively). We also show the equation of state parameters $w_{1}=w_{T}, w_{T e f f}$ (blue, yellow, respectively) and $w_{2}=w_{\phi}, w_{\phi e f f}$ (green, red, respectively) as a function of $N=\log [a]$. With these choice of $\lambda^{\prime} s$ the attractor solution has $\left(x_{1}, y_{1}\right)=(0.48,0.36)$ and $\left(x_{2}, y_{2}\right)=(0.48,0.34), \Omega_{1}=0.14, \Omega_{2}=0.35, \Omega_{b}=0.51$ and $w_{1 e f f}=w_{2 e f f}=w_{b}=0$.
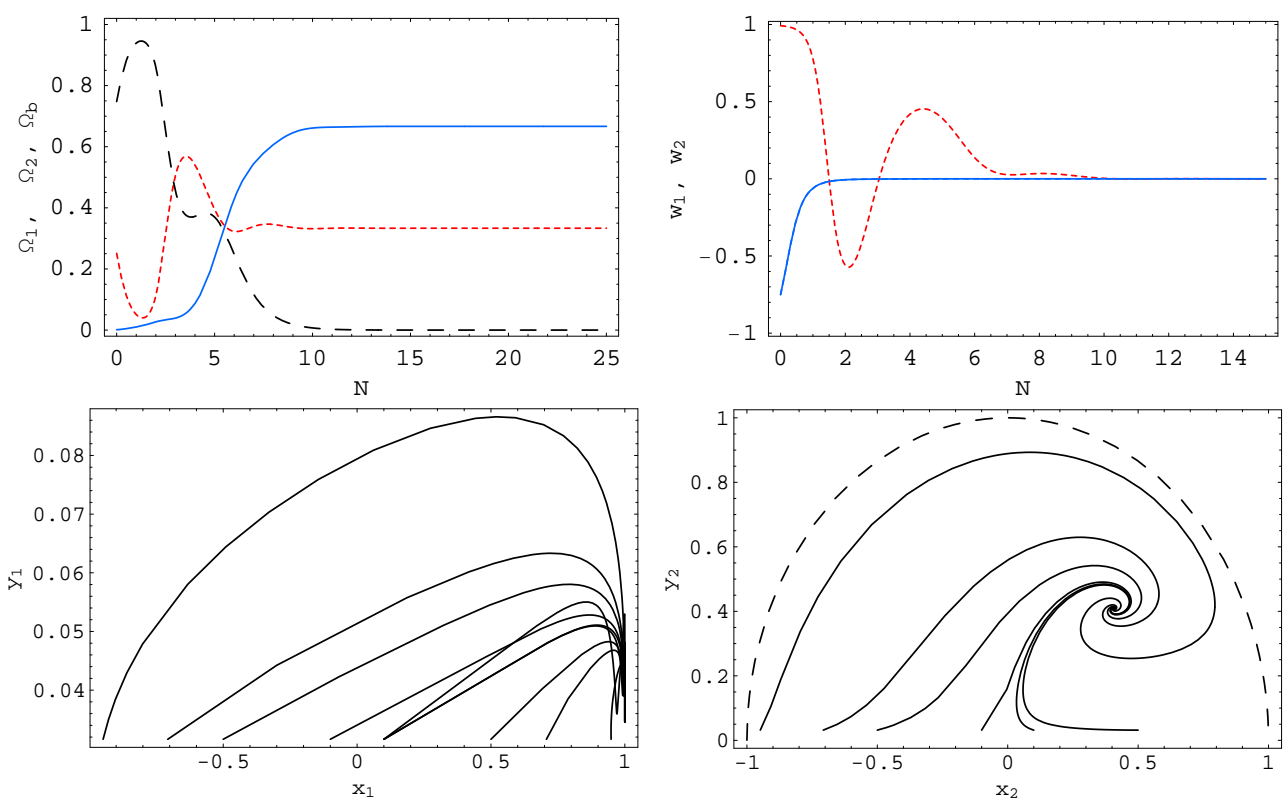

FIG. 2: We show for $\lambda_{1}=50, \lambda_{2}=0, \lambda_{3}=3$ and $\gamma_{b}=1+w_{b}=4 / 3$ the evolution of $\Omega_{1}=\Omega_{T}, \Omega_{2}=\Omega_{\phi}, \Omega_{b}$ (blue (solid), red (dotted) and black (dashed), respectively). We also show the equation of state parameters $w_{1}=w_{T}=w_{T e f f}($ blue) and $w_{2}=w_{\phi}=w_{\phi e f f}\left(\right.$ red (dotted)) as a function of $N=\log [a]$. With these choice of $\lambda^{\prime} s$ the attractor solution has $\left(x_{1}, y_{1}\right)=(0.99,0.03)$ and $\left(x_{2}, y_{2}\right)=(0.40,0.40), \Omega_{1}=2 / 3, \Omega_{2}=1 / 3, \Omega_{b}=0$ and $w_{1 e f f}=w_{2 e f f}=0$.

So, let us now determine under which conditions do both fields $T$ and $\phi$ have the same redshift. We see from 

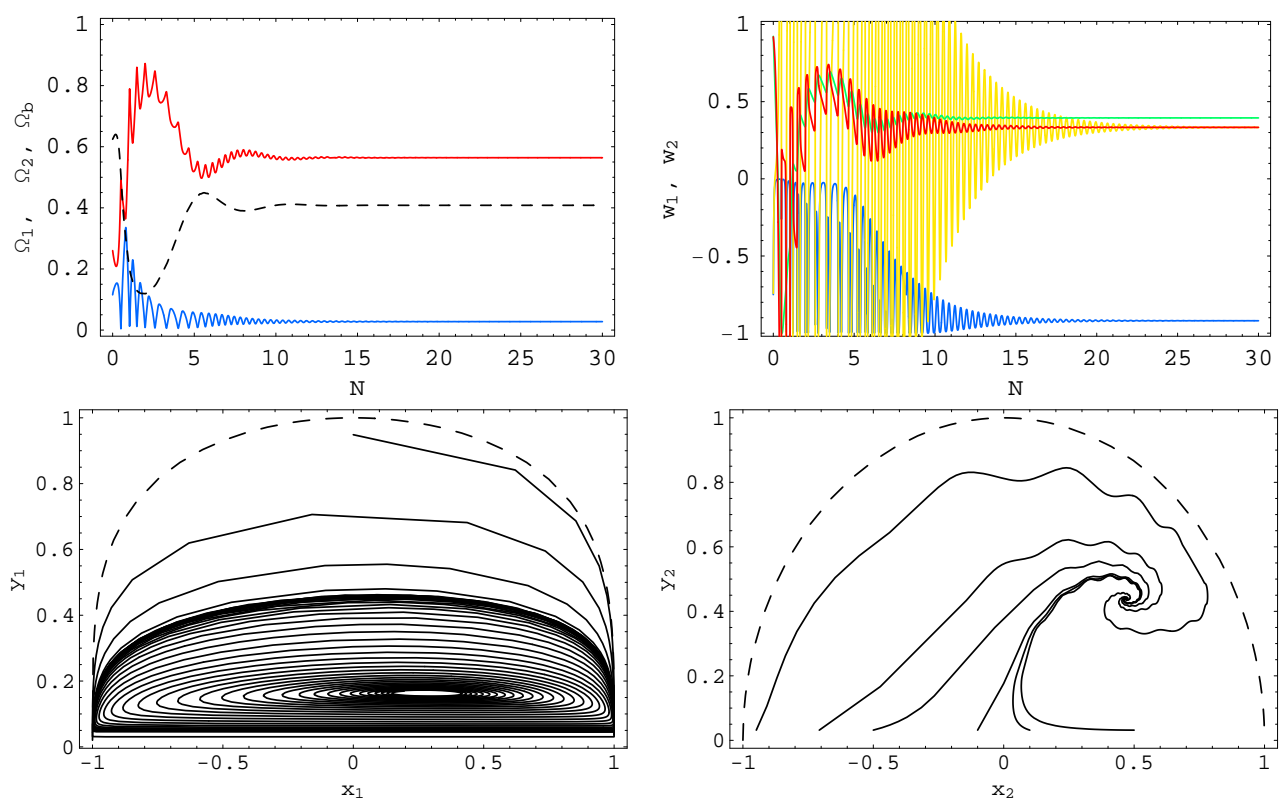

FIG. 3: We show for $\lambda_{1}=50, \lambda_{2}=-3, \lambda_{3}=3$ and $\gamma_{b}=1+w_{b}=4 / 3$ the evolution of $\Omega_{1}=\Omega_{T}, \Omega_{2}=\Omega_{\phi}, \Omega_{b}$ (blue (solid), red (dotted) and black (dashed), respectively). We also show the equation of state parameters $w_{1}=w_{T}, w_{T e f f}$ (blue, yellow, respectively) and $w_{2}=w_{\phi}, w_{\phi e f f}$ (green, red, respectively) as a function of $N=\log [a]$. With these choice of $\lambda^{\prime} s$ the attractor solution has $\left(x_{1}, y_{1}\right)=(0.28,0.16)$ and $\left(x_{2}, y_{2}\right)=(0.63,0.41), \Omega_{1}=0.03, \Omega_{2}=0.56, \Omega_{b}=0.41$ and $w_{1 e f f}=w_{2 e f f}=w_{b}=1 / 3$.
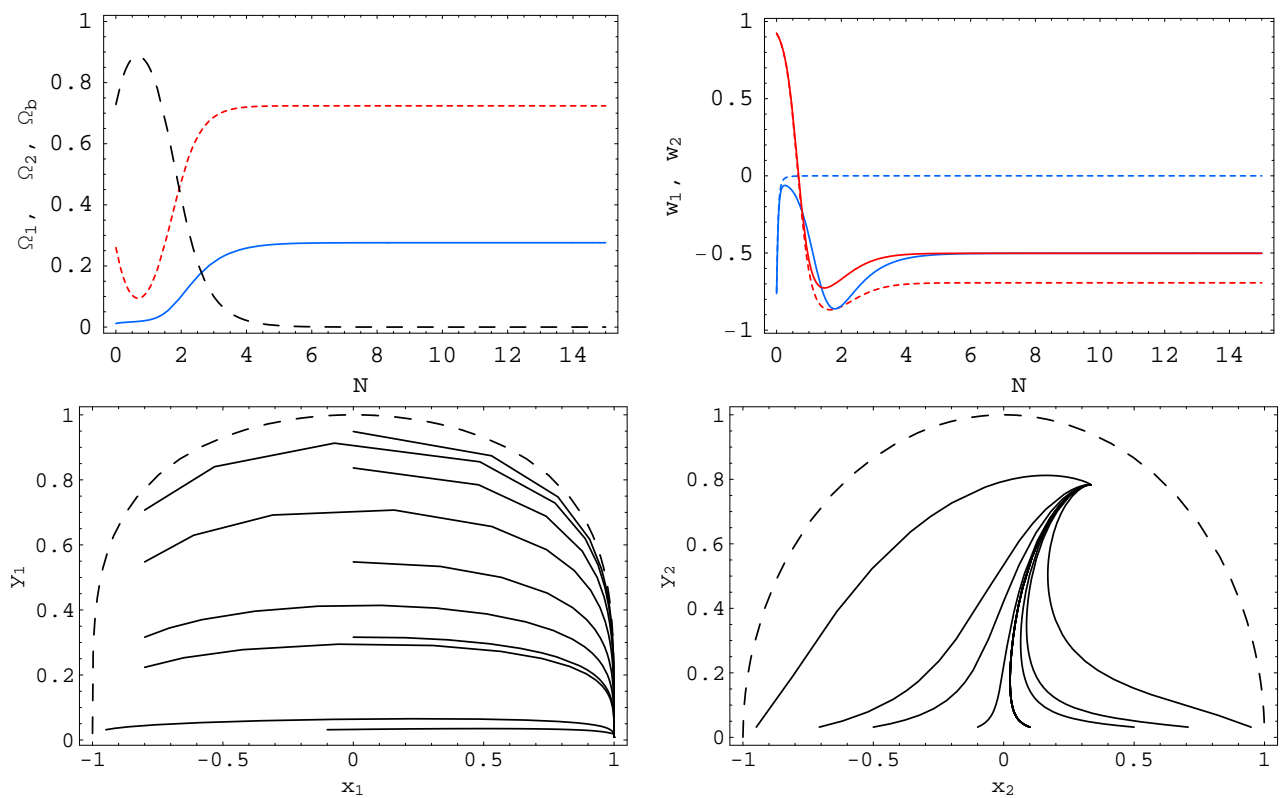

FIG. 4: We show for $\lambda_{1}=100, \lambda_{2}=1 / 2, \lambda_{3}=1$ and $\gamma_{b}=1+w_{b}=1$ the evolution of $\Omega_{1}=\Omega_{T}, \Omega_{2}=\Omega_{\phi}, \Omega_{b}$ (blue (solid), red (dotted) and black (dashed), respectively). We also show the equation of state parameters $w_{1}=w_{T}, w_{T e f f}$ (blue (dotted), blue (solid), respectively) and $w_{2}=w_{\phi}, w_{\phi e f f}\left(\right.$ red (dotted)), red (solid ), respectively). With these choice of $\lambda^{\prime} s$ the attractor solution has $\left(x_{1}, y_{1}\right)=(1,0)$ and $\left(x_{2}, y_{2}\right)=(1 / 3,0.78), \Omega_{1}=0.27, \Omega_{2}=0.73, \Omega_{b}=0$ and $w_{1 e f f}=w_{2 e f f}=w_{b}=-0.5$.

eqs.(32) that both effective equations of state are equal if

$$
\frac{\lambda_{2} x_{1} y_{2}^{3}}{\sqrt{3}}=\frac{\Omega_{1} \Omega_{2}}{\Omega_{1}+\Omega_{2}}\left(w_{1}-w_{2}\right)
$$



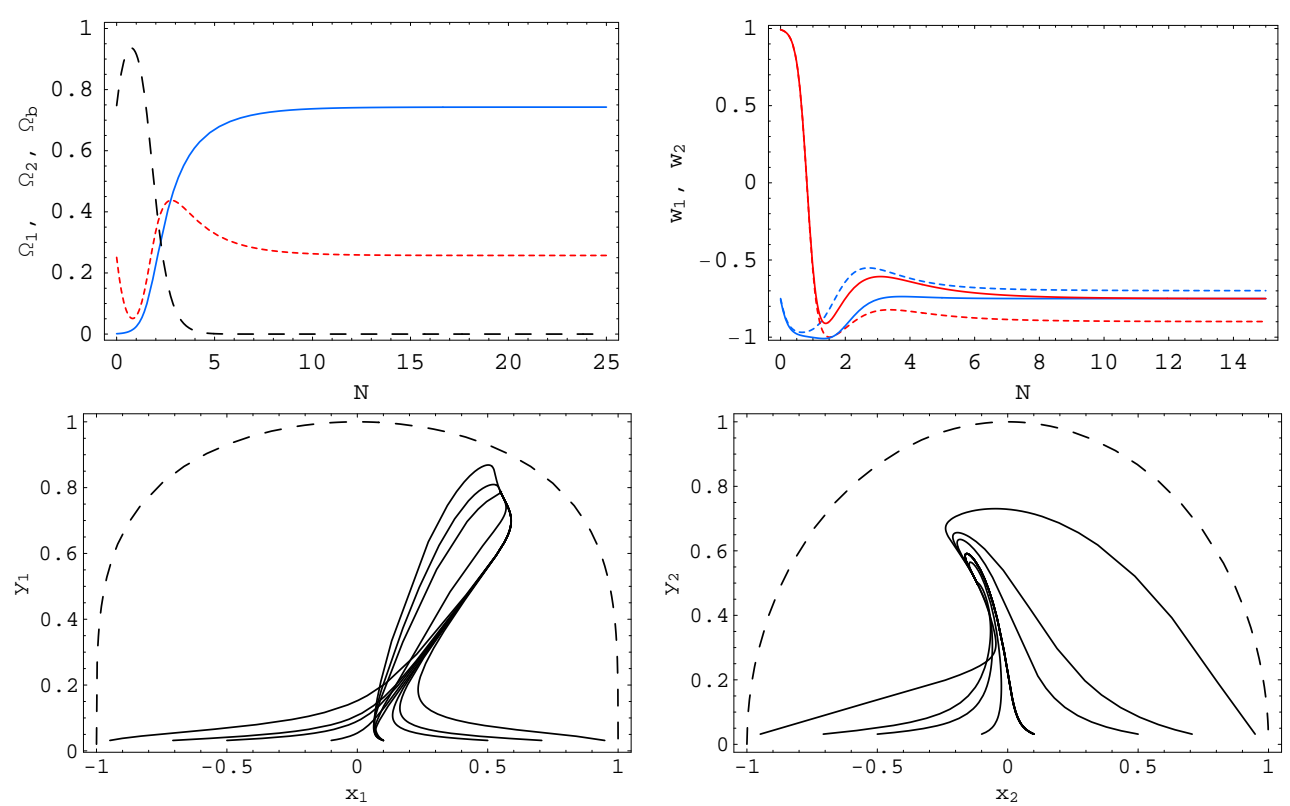

FIG. 5: We show for $\lambda_{1}=1, \lambda_{2}=1, \lambda_{3}=-1$ and $\gamma_{b}=1+w_{b}=1$ the evolution of $\Omega_{1}=\Omega_{T}, \Omega_{2}=\Omega_{\phi}, \Omega_{b}$ (blue (solid), red (dotted) and black (dashed), respectively). We also show the equation of state parameters $w_{1}=w_{T}, w_{T e f f}$ (blue, yellow, respectively) and $w_{2}=w_{\phi}, w_{\phi e f f}$ (green, red, respectively) as a function of $N=\log [a]$. With these choice of $\lambda^{\prime} s$ the attractor solution has $\left(x_{1}, y_{1}\right)=(0.55,0.8)$ and $\left(x_{2}, y_{2}\right)=(-0.11,0.49), \Omega_{1}=0.74, \Omega_{2}=0.26, \Omega_{b}=0$ and $w_{1 e f f}=w_{2 e f f}=-0.75$.

$$
=\Omega_{1}\left(\frac{3 H_{N}}{2 H}+\gamma_{1}\right)=-\Omega_{2}\left(\frac{3 H_{N}}{2 H}+\gamma_{2}\right)
$$

and using eq.(36) and eq.(37) we get

$$
\frac{\Omega_{1}}{\Omega_{2}}=\frac{\gamma_{1}-\gamma_{e f f}}{\gamma_{e f f}-\gamma_{2}}=\frac{w_{1}-w_{e f f}}{w_{e f f}-w_{2}}
$$

which coincides with eq.(19). The solution of the dynamical equations given in eqs.(24) for finite values of $x_{2}, y_{1}, y_{2}$ as a function of $x_{1}$ are

$$
\begin{aligned}
y_{1} & =\frac{\sqrt{3} \gamma_{e f f}}{\lambda_{1} x_{1}} \\
x_{2} & =\sqrt{\frac{2}{3}} \frac{\lambda_{3} y_{2}^{2}}{\left(2-\gamma_{e f f}\right)} \\
y_{2} & =\left(\frac{3 \sqrt{3} \gamma_{e f f}^{2}\left(x_{1}^{2}-\gamma_{e f f}\right)}{\lambda_{1}^{2} \lambda_{2} x_{1}^{3} \sqrt{1-x_{1}^{2}}}\right)^{1 / 3}=-\frac{\sqrt{3} \gamma_{e f f}^{2 / 3}}{\lambda_{1}^{2 / 3} \lambda_{2}^{1 / 3}} G\left(x_{1}\right) \\
x_{1} & =\frac{2}{\sqrt{3} \lambda_{2} y_{2}}\left(\frac{3 \gamma_{e f f}}{2}-\frac{\lambda_{3}^{2} y_{2}^{2}}{2-\gamma_{e f f}}\right)
\end{aligned}
$$

for $y_{1 N}=0, x_{2 N}=0, x_{1 N}=0, y_{2 N}=0$, respectively. We have defined

$$
G\left(x_{1}\right) \equiv \frac{1}{x_{1}}\left(\frac{\left(\gamma_{e f f}-x_{1}^{2}\right)}{\sqrt{1-x_{1}^{2}}}\right)^{1 / 3}
$$

and we obtain

$$
\Omega_{1}=\frac{3 \gamma_{e f f}^{2}}{\lambda_{1}^{2} x_{1}^{2} \sqrt{1-x_{1}^{2}}}
$$


where we have used eq.(39) in eq.(44) and (41). The values of $y_{1}, x_{2}, y_{2}$ are functions of $x_{1}$ and $\gamma_{e f f}$. Solutions with $\Omega_{1} \neq 0, \Omega_{2} \neq 0, \Omega_{b} \neq 0$ imply that $\gamma_{e f f}=\gamma_{b}=\gamma_{1 \text { eff }}=\gamma_{2}$ eff is a given constant (given by the barotropic fluid). If however, $\gamma_{e f f}=\gamma_{1 \text { eff }}=\gamma_{2}$ eff $<\gamma_{b}$ then $\Omega_{b}=0$ and $\gamma_{\text {eff } f}$ is not a constant given a priori but it is a constant which is a function of $x_{i}, y_{i}$. In this case eqs.(39)-(42) must be supplemented with eq.(36). Using eqs.(39)-(41) we can rewrite eq.(36) as

$$
1=\frac{\Omega_{1} \gamma_{1}}{\gamma_{\text {eff }}}+\frac{\Omega_{2} \gamma_{2}}{\gamma_{\text {eff }}}=\frac{3 \gamma_{\text {eff }}}{\lambda_{1}^{2} \sqrt{1-x_{1}^{2}}}+\frac{3^{3} 4 \lambda_{3}^{2} \gamma_{e f f}^{5 / 3}}{(2-\gamma)^{2}} G\left(x_{1}\right)^{4}
$$

which is only valid if $\Omega_{b}=0$.

Let us take $\gamma_{e f f}=\gamma_{b}$ with a barotropic fluid with $\gamma_{b} \geq 1$, i.e. it includes matter $\gamma_{b}=1$ and radiation $\gamma_{b}=4 / 3$, and in this case we get the following constraints on $\lambda_{1}, \lambda_{2}, \lambda_{3}$, from requiring $\left|x_{i}\right|<1$ and $0<y_{1}<1$ :

$$
\begin{aligned}
\lambda_{1}^{2} & >\frac{3 \gamma_{e f f}^{2}}{x_{1}^{2} \sqrt{1-x_{1}^{2}}}>\frac{9 \sqrt{3} \gamma_{e f f}^{2}}{2} \\
\left|\lambda_{1}^{2} \lambda_{2}\right| & >\left|\frac{3 \sqrt{3} \gamma_{e f f}^{2}\left(\gamma_{e f f}-x_{1}^{2}\right)}{x_{1}^{3} \sqrt{1-x_{1}^{2}}}\right|>3 \sqrt{3} \gamma_{e f f}^{2} G_{m}^{3} \\
\lambda_{2} x_{1} & <0, \quad \lambda_{1} x_{1}>0, \quad \lambda_{3} x_{2}>0, \quad \lambda_{1} \lambda_{2}<0 \\
\frac{\lambda_{1}^{4 / 3} \lambda_{2}^{2 / 3}\left(2-\gamma_{e f f}\right) \alpha}{2 \gamma_{e f f}^{1 / 3} G_{m}^{2}} & >\lambda_{3}^{2}>\frac{3 \gamma_{e f f}\left(2-\gamma_{e f f}\right) \alpha}{2}
\end{aligned}
$$

where $G_{m}$ in eqs.(47) and (49) is the minimum value of $G\left(x_{1}\right)$. The constraint (46) comes from requiring $\Omega_{1}<1$ and $\left(x_{1}^{2} \sqrt{1-x_{1}^{2}}\right)^{-1}$ has a minimum value $\left(x_{1}^{2} \sqrt{1-x_{1}^{2}}\right)^{-1}=3 \sqrt{3} / 2$ at $x_{1}^{2}=2 / 3$. Eq.(47) comes from eq.(41) and $0<y_{2}<1$. Eq.(48) arises from requiring $0<y_{1}$ and $0<y_{2}$ and we see from eq.(41) that $G \lambda_{2}$ must be negative, which for $\gamma_{e f f} \geq 1$ implies that $\lambda_{2} x_{1}<0$. Finally eq.(49) comes from eq.(50) and $0<y_{2}<1$.

For $\gamma_{b}=\gamma_{e f f}=1$ the function $G$ is simply $G=\left(1-x_{1}^{2}\right)^{1 / 6} / x_{1}$ and $G$ takes the value $|G(0)|=\infty$ and $G( \pm 1)=$ $G_{m}=0$ so that $0 \leq|G(x)| \leq \infty$. Therefore, the lower and upper constraints in eq.(47) and (49), respectively, do not impose any limitation to $\lambda_{i}$. On the other hand if $\gamma_{b}=\gamma_{e f f}>1$ (as for radiation $\gamma_{b}=4 / 3$ ) then $|G( \pm 1)|=|G(0)|=\infty$ and $G(x)$ has a minimum absolute value $G_{m} \equiv\left|G\left(x=x_{m}\right)\right|$ at $x_{m}^{2}=\left[1+4 \gamma_{\text {eff }}-\sqrt{1+16 \gamma_{e f f}\left(\gamma_{e f f}-1\right)}\right] / 2$, e.g. for $\gamma_{e f f}=4 / 3$ we have $x_{m}=0.93$ and $G_{m}=1.16$. The fact that $G$ has a minimum value for $\gamma_{e f f}=\gamma_{b}>1$ implies a constraint on the value of $\lambda_{1}, \lambda_{2}$ namely $\lambda_{1}^{2} \lambda_{2}>3 \sqrt{3} \gamma_{e f f}^{2} G_{m}^{3}$ as seen from eq.(47) and from eq.(49) an upper value for $\lambda_{3}$.

To finally solve eqs.(39)-(42) we substitute $y_{2}$ from eq.(41) into eq.(42) and we get a nonlinear equation for $x_{1}$ only

$$
y_{2}^{2}=\frac{3 \gamma_{e f f}^{4 / 3}}{\lambda_{1}^{4 / 3} \lambda_{2}^{4 / 3}} G\left(x_{1}\right)^{2}=\frac{3\left(2-\gamma_{e f f}\right)}{2 \lambda_{3}^{2}}\left(\gamma_{e f f}-\frac{\lambda_{2} x_{1} y_{2}}{\sqrt{3}}\right) \equiv \frac{3\left(2-\gamma_{e f f}\right) \gamma_{e f f} \alpha}{2 \lambda_{3}^{2}}
$$

with

$$
\alpha \equiv 1+\left(\frac{\lambda_{2}}{\lambda_{1}}\right)^{2 / 3} \gamma_{e f f}^{-1 / 3} x_{1} G\left(x_{1}\right)
$$

and $\alpha$ depends on $\lambda_{2} / \lambda_{1}$ and $x_{1}$ with $\alpha>1$ since the second term in eq.(51) is positive for $\gamma_{e f f} \geq 1$. Eq.(50) is however valid for any value of $\gamma_{e f f}$ including $\gamma_{e f f}<1$. In this later case the second term in eq.(51) is no longer positive definite $\left(x_{1} G\right.$ is negative for $\left.x_{1}^{2}>\gamma_{e f f}\right)$ and the fact that $\alpha$ must be non negative since $y_{2}^{2}>0$, gives the constraint $\lambda_{2}^{2 / 3} \lambda_{1}^{-2 / 3} \gamma_{\text {eff }}^{-1 / 3} x_{1} G\left(x_{1}\right)>-1$.

The solution to eq.(50) is non analytical and must be solved numerically for $x_{1}$. In terms of $G$ eq.(50) gives

$$
G\left(x_{1}\right)^{2}=\frac{\lambda_{1}^{4 / 3} \lambda_{2}^{2 / 3}}{\lambda_{3}^{2}} \frac{\alpha\left(2-\gamma_{e f f}\right)}{2 \gamma_{e f f}^{1 / 3}} \geq G_{m}^{2}
$$

For $\gamma_{b}=\gamma_{e f f}=1$ eq.(52) does not impose a constraint on the choice of $\lambda_{1}, \lambda_{2}, \lambda_{3}$ since $G$ can take values form zero to infinity and $G_{m}=0$. However, for $\gamma_{e f f}>1$ the function $G$ has a minimum value given by $G_{m}$, therefore the choice of $\lambda_{1}, \lambda_{2}, \lambda_{3}$ must be such as to give a value of $G$ larger than $G_{m}$ and for $\gamma_{b}=4 / 3$, as mentioned before, we have $G_{m}=1.16$. 
Regarding the quantity $\alpha$ given in eq.(51) which term dominates (first or second) depends on the value of $\lambda_{2} / \lambda_{1}$ and $x_{1}$ through the function $F\left(x_{1}\right)=x_{1} G\left(x_{1}\right)=\left(\left(\gamma_{e f f}-x_{1}^{2}\right) / \sqrt{1-x_{1}^{2}}\right)^{1 / 3}$. The function $F$ has a minimum value at $x_{1}=2-\gamma_{\text {eff }}$ giving a value $\left.F\right|_{m}=2^{1 / 3}\left(\gamma_{\text {eff }}-1\right)^{1 / 6}$. For $\gamma_{\text {eff }}=1$ one has $0 \leq F=\sqrt{1-x_{1}^{2}} \leq 1$ while for $\gamma_{\text {eff }}>1$ one finds $\left.F\right|_{m} \leq F \leq F(x=1)=\infty$. However, since $F(0)=F\left(\widetilde{x}_{1}\right)=\gamma_{\text {eff }}^{1 / 3}$ for $\widetilde{x}_{1}=\sqrt{\gamma_{e f f}\left(2-\gamma_{e f f}\right)}$ the value of $F$ in the case $\gamma_{e f f}>1$ is constraint between $F_{m} \leq F<F(0)=F\left(\widetilde{x}_{1}\right)=\gamma_{\text {eff }}^{1 / 3}$ for $0<\left|x_{1}\right|<\sqrt{\gamma_{e f f}\left(2-\gamma_{e f f}\right)}$, e.g. for $\gamma_{\text {eff }}=4 / 3$ we have $F_{m}=1.05 \leq F \leq F(0)=(4 / 3)^{1 / 3}=1.1$ for $0<\left|x_{1}\right|<2 \sqrt{2} / 3=.94$ which covers most of the range $\left|x_{1}\right|=(0,1)$. Therefore, $\gamma_{e f f}^{-1 / 3} F$ is in general of order unity giving $\alpha$ in eq.(51) as

$$
\alpha \simeq 1+\left(\lambda_{2} / \lambda_{1}\right)^{2 / 3} .
$$

If we have $\gamma_{e f f}=\gamma_{1 \text { eff }}=\gamma_{2}$ eff $<\gamma_{b}$ then $\Omega_{b}=0$ and $\gamma_{e f f}$ is not a given constant but is a function of $x_{i}, y_{i}$ and may be smaller than 1. In this case the solution to eqs.(39)-(42) is given by solving eqs.(45) and (52) numerically. If $\gamma_{e f f}<1$ then the minimum of $|G|$ and $|F|$ are $G_{m}=F_{m}=0$ with $0 \leq G \leq \infty$ and $0 \leq F \leq \infty$ and there is no upper constraint on $\lambda_{3}$ from eq.(49) and no lower constrain on $\left|\lambda_{1}^{1} \lambda_{2}\right|$ in eq.(47). However, since $F\left(x_{1}\right)=x_{1} G\left(x_{1}\right)=$ $\left(\left(\gamma_{e f f}-x_{1}^{2}\right) / \sqrt{1-x_{1}^{2}}\right)^{1 / 3}$ can now be negative for $x_{1}^{2}>\gamma_{\text {eff }}$ the condition $y_{2}>0$ (i.e. $\left.\alpha>0\right)$ implies from eq. (51) the constraint

$$
F\left(x_{1}\right)^{3}=\frac{\gamma_{e f f}-x_{1}^{2}}{\sqrt{1-x_{1}^{2}}}>-\frac{\lambda_{1}^{2} \gamma_{e f f}}{\lambda_{2}^{2}}
$$

\section{ASYMPTOTIC BEHAVIOR}

Special cases can be studied by taking different limits of the parameters $\lambda_{i}$. A constant $\lambda_{1}$ is given by a potential $V=V_{o} / T^{2}$ and we have $\lambda_{1}=2 / \sqrt{V_{o}}$. A constant $\lambda_{3}$ can be obtained for example if we consider a factorizeable interaction potential $B(\phi, \varphi)=h(T) K(\phi)$. In this case $\lambda_{3}=-K_{\phi} / K$ and taking $K(\phi)$ as an exponential potential we have $\lambda_{3}=-\beta$ for $K=K_{o} e^{\beta \phi}$. However, a factorizeable potential $B$ gives $\lambda_{2}=-h_{T} /\left(h^{3 / 2} K^{1 / 2}\right)$ which is now a function of both fields $T$ and $\phi$. So, a constant $\lambda_{2}$ can only be obtained as a combination of the evolution of $T, \phi$ and cannot be trivially anticipated just by looking at the functional form of $B$.

An interesting limit is when $x_{1}^{2}=\dot{T}^{2}=1$. In this case $y_{1}=0$ since $\Omega_{1}=y_{1}^{2} / \sqrt{1-x_{1}^{2}}$ must be smaller than one. The value of $\Omega_{1}$ depends on the value of the parameters $\lambda_{i}$. Solving eqs.(24) we find two solutions. One solution has $x_{1}^{2}=1, y_{1}=0$ and

$$
\begin{aligned}
& x_{2}=\sqrt{\frac{3}{2}} \frac{1}{4 \lambda_{3}^{2}}\left(\left(2-\gamma_{e f f}\right) \lambda_{2}^{2}+4 \gamma_{e f f} \lambda_{3}^{2}-\lambda_{2} \operatorname{sgn}\left[x_{1}\right] \sqrt{8\left(2-\gamma_{e f f}\right) \gamma_{e f f} \lambda_{3}^{2}+\left(2-\gamma_{e f f}\right)^{2} \lambda_{2}^{2}}\right) \\
& y_{2}=\frac{\sqrt{3}}{4 \lambda_{3}^{2}}\left(-\left(2-\gamma_{e f f}\right) \lambda_{2} \operatorname{sgn}\left[x_{1}\right]+\sqrt{8\left(2-\gamma_{e f f}\right) \gamma_{e f f} \lambda_{3}^{2}+\left(2-\gamma_{e f f}\right)^{2} \lambda_{2}^{2}}\right)
\end{aligned}
$$

where $\operatorname{sgn}\left[x_{1}\right]= \pm 1$ and eqs.(55) constrains the values of $\lambda_{2}, \lambda_{3}$ since $\left|x_{2}\right|<1,0<y_{2}<1$. We see that this solution requires $\left|\lambda_{2} / \lambda_{3}\right|<1$. The second solution has $x_{1}^{2}=1, y_{1}=0$ and $\left(x_{2}, y_{2}\right)=(0,0)$, i.e. $\Omega_{2}=0$, which reduce to the case without canonical scalar field discussed below.

In the limit $\lambda_{2}=0$ the eqs.(24) reduce to two uncoupled scalar fields with a vanishing interaction term $\delta$ and $\gamma_{1 \text { eff }}=\gamma_{1}, \gamma_{2}$ eff $=\gamma_{2}$. The solution can be obtain from the combination of the work given in [6] and [3], where a tachyon field and a scalar field in the presence of a barotropic fluid, respectively, are studied. Which term dominates at late times depends on which equation of state is smaller and this depends on the values of $\lambda_{1}, \lambda_{3}$. The equation of state for the field $T$ is given by $\gamma_{1 \text { eff }}=\gamma_{1}=\left(\sqrt{\lambda_{1}^{4}+36}-\lambda_{1}^{2}\right) \lambda_{1}^{2} / 18$ if $\gamma_{b}>\gamma_{1}$ and $\gamma_{1 \text { eff }}=\gamma_{1}=\gamma_{b}$ otherwise [6] while for the field $\phi$ we have $\gamma_{2}$ eff $=\gamma_{2}=\lambda_{3}^{2} / 3$ for $\lambda_{3}^{2}<3 \gamma_{b}$ and $\gamma_{2}$ eff $=\gamma_{2}=\gamma_{b}$ if $\lambda_{3}^{2}>3 \gamma_{b}$ [3]. Therefore if $\lambda_{3}^{2}<\left(\sqrt{\lambda_{1}^{4}+36}-\lambda_{1}^{2}\right) \lambda_{1}^{2} / 6$ then we have $\gamma_{2}<\gamma_{1}$ and the energy density $\rho_{\phi}$ will dominate over $\rho_{T}$ at late times. For $\lambda_{3}^{2}>\left(\sqrt{\lambda_{1}^{4}+36}-\lambda_{1}^{2}\right) \lambda_{1}^{2} / 6$ we have $\gamma_{2}>\gamma_{1}$ and the energy density $\rho_{T}$ will dominate over $\rho_{\phi}$.

In the limit $\lambda_{3}=0$ we have from eqs.(24) $x_{2 N} / x_{2}<0$ which implies a decreasing $x_{2}$ with an asymptotic limit $x_{2}=0$. In this case eqs.(24) or equivalently eq.(50) the solution requires $\alpha=0$, i.e. $F\left(x_{1}\right)=x_{1} G\left(x_{1}\right)=-\left(\lambda_{1} / \lambda_{2}\right)^{2 / 3} \gamma_{\text {eff }}^{1 / 3}$. Once $x_{1}$ has been obtained from $\alpha=0$ we can use eqs.(39) and (41) to obtain the values of $y_{1}$ and $y_{2}$.

Finally, if we take $\lambda_{1}=0$ then from $y_{1 N}=0$ in eqs.(24) we have $y_{1} H_{N} / H=0$. If $H_{N} / H=0$ then $x_{2}=x_{1}=\Omega_{b}=0$ and from $x_{2 N}=0$ we conclude that $y_{2}=0$ and the universe is dominate by a constant tachyonic potential $\Omega_{1}=y_{1}^{2}=1$. If however, $y_{1}=0$ then from $x_{1 N}=0$ we need $x_{1}^{2}=1$ or $y_{2}=0$. For $x_{1}^{2} \neq 1$ the solution has $y_{1}=y_{2}=x_{2}=0$ with $\Omega_{1}=\Omega_{2}=0$ and $\Omega_{b}=1$. If $x_{1}^{2}=1$ the solution is $y_{1}=0$ and $x_{2}, y_{2}$ are given in eqs.(55). 


\section{A. Particle Physics Model}

Let us now take a specific choice of potentials motivated by particle physics. We consider a factorizeable interaction potential

$$
B(\phi, \varphi)=h(T) K(\phi)
$$

In this case the $\lambda_{i}$ parameters become only functions of a single field

$$
\lambda_{1}=-\frac{V_{T}}{V^{3 / 2}}, \quad \lambda_{2}=-\frac{h_{T}}{h^{3 / 2} K^{1 / 2}}, \quad \lambda_{3}=-\frac{K_{\phi}}{K} .
$$

We see from eq.(57) that $\lambda_{1}$ is only a function of $T, \lambda_{3}$ is only a function of $\phi$ while $\lambda_{2}$ depends on $T$ and $\phi$. Let us take from eq.(11) $d V_{\text {eff }} / d T=\left(V_{T}+B_{T} \sqrt{1-\dot{T}^{2}}\right) V / \rho_{T}^{2}=0$. This equation has two solutions given in eqs.(12) and (13). If $\dot{T} \neq 1$ then using the anstaz in eq.(56) we have from eq.(11)

$$
K(\phi)=-\frac{V_{T}}{h_{T} \sqrt{1-\dot{T}^{2}}}=-\frac{\rho_{T} V_{T}}{h_{T} V}
$$

where we have used $\rho_{T}=V / \sqrt{1-\dot{T}^{2}}$ and substituting $K$ in eq.(57) we get

$$
\lambda_{2}=\left(\frac{h_{T}}{h}\right)^{3 / 2}\left(-\frac{V}{V_{T}}\right)^{1 / 2} \frac{1}{\rho_{T}^{1 / 2}} .
$$

The solution in eq.(13) has $\dot{T} \simeq 0$ and $\rho_{T} \simeq V$. As an example let us take the potential for brane-tachyon field as $V=V_{o} e^{-T^{2} / 2}$ and the interaction term as $B=B_{o} e^{\beta T} \phi^{n}$, i.e. $h=e^{\beta T}$ and $K=B_{o} \phi^{n}$, with $V_{o}>0, B_{o}>0$. In this case we have from eq.(58) $K=\rho_{T} T e^{-\beta T} / \beta$

$$
\lambda_{1}=T e^{T^{2} / 4}, \quad \lambda_{2}=-\frac{\beta^{3 / 2}}{\left(T \rho_{T}\right)^{1 / 2}}, \quad \lambda_{3}=-\frac{n}{\phi}= \pm n K^{-1 / n}= \pm \frac{n \beta^{1 / n} e^{\beta T / n}}{\left(T \rho_{T}\right)^{1 / n}}
$$

and in the limit $T \rightarrow \infty$ we have

$$
\lambda_{1} \rightarrow \infty, \quad \lambda_{2} \rightarrow-\infty, \quad \lambda_{3} \rightarrow \pm \infty
$$

where we have taken $\beta>0$ as required by eq.(11) since $V_{T}$ and $B_{T}$ must have opposite signs. The relative growth is given by

$$
\frac{\lambda_{1}}{\lambda_{2}} \simeq T^{3 / 2}, \quad \frac{\lambda_{3}}{\lambda_{1}} \simeq T^{-(n+1) / n} e^{\beta T+T^{2}(2-n) / 4 n}, \quad \frac{\lambda_{3}}{\lambda_{2}} \simeq T^{(n-2) / n} e^{\beta T+T^{2}(2-n) / 4 n}
$$

Form eq.(62) we have $\lambda_{1} \gg\left|\lambda_{2}\right|$ and for $n=2$ we get $\left|\lambda_{3}\right| \gg \lambda_{1} \gg\left|\lambda_{2}\right|$ while for $n>2$ we find $\lambda_{1} \gg\left|\lambda_{2}\right| \gg\left|\lambda_{3}\right|$. On the other hand if $\dot{T}^{2}=1$ then we have $V=0$ and the solution has either $\Omega_{2}=0$ or it is given by eq.(55). Solving numerically eqs.(2) and (3) with $n=4, \beta=1, \gamma_{b}=1$ we find $\dot{T}^{2} \rightarrow 1$ with $\left|\lambda_{1}\right| \gg\left|\lambda_{2}\right| \gg\left|\lambda_{3}\right| \gg 1$ and $\Omega_{2}=\Omega_{b}=0$.

\section{B. Examples}

We now present four different attractor solution depending on the values of $\lambda_{1}, \lambda_{2}, \lambda_{3}$. We show in figures 1-5 the evolution of $\Omega_{\phi} \equiv \Omega_{1}, \Omega_{\varphi} \equiv \Omega_{2}, \Omega_{b}$ and $w_{1} \equiv w_{\phi}, w_{\phi e f f}, w_{2} \equiv w_{\varphi}, w_{\varphi e f f}$ for the different choices of $\lambda^{\prime} s$. We also show the phase space of $\left(x_{1}, y_{1}\right)$ and $\left(x_{2}, y_{2}\right)$ for each case. Since the phase space depends on four variables, namely $\left(x_{1}, y_{1}, x_{2}, y_{2}\right)$, it is no surprising that the curves in the two dimensional space $\left(x_{1}, y_{1}\right)$ and $\left(x_{2}, y_{2}\right)$ may cross.

In figs. 1 we have $\lambda_{1}=10, \lambda_{2}=-10, \lambda_{3}=-5$ and $\gamma_{b}=1+w_{b}=1$. The conditions in eqs.(46)-(49) are therefore satisfied and we expect to have $\Omega_{1}, \Omega_{2}, \Omega_{b}$ different than zero. In fact we get $\Omega_{1}=0.14, \Omega_{2}=0.35, \Omega_{b}=0.51$ and $\left(x_{1}, y_{1}\right)=(0.48,0.36)$ and $\left(x_{2}, y_{2}\right)=(0.48,0.34)$. The effective equations of state are $w_{1 e f f}=w_{2 e f f}=w_{b}=0$ giving a decelerating universe.

In figs. 2 we show a model with vanishing interaction term $\delta=0$, i.e. $\lambda_{2}=0$, and with $\lambda_{1}=50, \lambda_{3}=3$ and $\gamma_{b}=$ $1+w_{b}=4 / 3$. The attractor solution has $\Omega_{1}=2 / 3, \Omega_{2}=1 / 3, \Omega_{b}=0$ and $\left(x_{1}, y_{1}\right)=(0.99,0.03)\left(x_{2}, y_{2}\right)=(0.40,0.40)$. 
The equation of state $w_{i}$ is equal to the effective equation of state $w_{i e f f}=w_{i}=0$ and $T$ redshifts as matter at late times.

In figs. 3 we show the same model as in figs. 2 but with an interaction term $\delta \neq 0$. We take $\lambda_{2}=-3$ and $\lambda_{1}=50, \lambda_{3}=$ 3 and $\gamma_{b}=1+w_{b}=4 / 3$. The attractor solution has $\Omega_{1}=0.03, \Omega_{2}=0.56, \Omega_{b}=0.41$ and $\left(x_{1}, y_{1}\right)=(0.28,0.16)$ $\left(x_{2}, y_{2}\right)=(0.63,0.41)$. The effective equation of state is given by $w_{1 \text { eff }}=w_{2 \text { eff }}=w_{b}=1 / 3$ which shows that the tachyon field and $\phi$ redshift as radiation. We see that the relevance of the interaction term in figs.3 which makes $w_{1 e f f}$ go from $w_{1 e f f}=0$ (no interaction) to $w_{1 e f f}=1 / 3$ (with interaction). This shows that an interaction term can solve the problem of the tachyon field redshifting as matter and dominating the universe well before radiation-matter equality.

In figs. 4 we have $\lambda_{1}=100, \lambda_{2}=1 / 2, \lambda_{3}=1$ and $\gamma_{b}=1+w_{b}=1$. With these choice of $\lambda^{\prime} s$ the attractor solution has $\left(x_{1}, y_{1}\right)=(1,0)$ and $\left(x_{2}, y_{2}\right)=(1 / 3,0.78), \Omega_{1}=0.27, \Omega_{2}=0.73, \Omega_{b}=0$ and $w_{1 e f f}=w_{2 e f f}=w_{b}=-0.5$. Notice that even tough $y_{1} \rightarrow 0$ we have $x_{1} \rightarrow 1$ and a finite $\Omega_{1}$ which dominates the universe with $w_{1 e f f}=w_{2 e f f}=-0.5$ giving a positive acceleration. Notice that $w_{1} \rightarrow 0$ as for a tachyon with no interaction, however the interaction term gives a negative effective equation of state.

In figs. 5 we have $\lambda_{1}=1, \lambda_{2}=1, \lambda_{3}=-1$ and $\gamma_{b}=1+w_{b}=1$. The conditions in eqs.(46)-(49) are therefore not satisfied and we do not expect to have $\Omega_{1}, \Omega_{2}, \Omega_{b}$ different than zero. In fact we get $\Omega_{1}=0.74, \Omega_{2}=0.26, \Omega_{b}=0$, i.e. the $T$ field dominates at late times, and $\left(x_{1}, y_{1}\right)=(0.55,0.8)\left(x_{2}, y_{2}\right)=(-0.11,0.49)$. The effective equations of state are $w_{1 e f f}=w_{2 e f f}=-0.75$ giving an accelerating universe.

\section{SUMMARY AND CONCLUSIONS}

We have studied the dynamical system of two scalar fields, a tachyon and a canonically normalized field, with arbitrary potentials in the presence of a barotropic fluid in a FRW metric. We have shown that all model dependence is given in terms of three parameters, namely $\lambda_{1}=-V_{T} / V^{3 / 2}, \lambda_{2}=-B_{T} / B^{3 / 2}$ and $\lambda_{3}=-B_{\phi} / B$. The solution to the dynamical equations given in (24) are non linear and general analytic solution does not exist. If one of the scalar fields dominates over the other one then eqs.(24) will be reduced to a single scalar field, either $T$ or $\phi$, in the presence of a barotropic fluid. These special cases have been studied in the literature, for a tachyon domination in [6] and for a canonical scalar field in [3]. We have determined the critical points in the case where the two scalar fields $T$ and $\phi$ have the same redshift. The solution is a function of the parameters $\lambda_{i}$ and we have calculated the restrictions on these parameters. We have solved numerically for different interesting cases and we have shown that either scalar field or the barotropic fluid can dominate at late times. The interaction term between the tachyon and the scalar field changes the effective equation of state for $T$ and it is possible for a tachyon field to redshift as matter in the absence of an interaction term $B$ and as radiation when $B$ is turned on. This results solves then the tachyonic matter problem.

\section{Acknowledgments}

This work was also supported in part by CONACYT project 45178-F and DGAPA, UNAM project IN114903-3.

[1] A. Sen, JHEP 0204, 048 (2002); A. Sen, JHEP 0207, 065 (2002); A. Sen, Mod. Phys. Lett. A 17, 1797 (2002); A. Sen, Phys. Scripta T117, 70 (2005); A. Sen, JHEP 9910, 008 (1999); M. R. Garousi, Nucl. Phys. B 584, 284 (2000); M. R. Garousi, Nucl. Phys. B 647, 117 (2002); M. R. Garousi, JHEP 0305, 058 (2003); E. A. Bergshoeff, M. de Roo, T. C. de Wit, E. Eyras and S. Panda, JHEP 0005, 009 (2000); J. Kluson, Phys. Rev. D 62, 126003 (2000); D. Kutasov and V. Niarchos, Nucl. Phys. B 666, 56 (2003);

[2] G. W. Gibbons, Class. Quant. Grav. 20, S321 (2003); G. W. Gibbons, Phys. Lett. B 537, 1 (2002); M. Fairbairn and M. H. G. Tytgat, Phys. Lett. B 546, 1 (2002); A. Feinstein, Phys. Rev. D 66, 063511 (2002); S. Mukohyama, Phys. Rev. D 66, 024009 (2002) D. Choudhury, D. Ghoshal, D. P. Jatkar and S. Panda, Phys. Lett. B 544, 231 (2002); G. Shiu and I. Wasserman, Phys. Lett. B 541, 6 (2002); L. Kofman and A. Linde, JHEP 0207, 004 (2002); M. Sami, Mod. Phys. Lett. A 18, 691 (2003); G. N. Felder, L. Kofman and A. Starobinsky, JHEP 0209, 026 (2002); S. Mukohyama, Phys. Rev. D 66, 123512 (2002); M. C. Bento, O. Bertolami and A. A. Sen, Phys. Rev. D 67, 063511 (2003); J. G. Hao and X. Z. Li, Phys. Rev. D 66, 087301 (2002); C. J. Kim, H. B. Kim and Y. B. Kim, Phys. Lett. B 552, 111 (2003); B. C. Paul and M. Sami, Phys. Rev. D 70, 027301 (2004); T. Padmanabhan, Phys. Rev. D 66, 021301 (2002); J. S. Bagla, H. K. Jassal and T. Padmanabhan, Phys. Rev. D 67, 063504 (2003); A. V. Frolov, L. Kofman and A. A. Starobinsky, Phys. Lett. B 545, 8 (2002); 
[3] A. de la Macorra, G. Piccinelli, Phys.Rev.D61:123503,2000.

[4] T. Barreiro, Edmund J. Copeland, N.J. Nunes, Phys.Rev.D61:127301,2000.

[5] A. de la Macorra, U. Filobello, G. German, Phys.Lett.B635:355-363,2006.

[6] E. J. Copeland, M. R. Garousi, M. Sami and S. Tsujikawa, Phys. Rev. D 71, 043003 (2005); J.M. Aguirregabiria, R. Lazkoz, Phys.Rev.D69:123502,2004

[7] A. de la Macorra astro-ph/0703702 